\title{
Standards für Ethikberatung in Einrichtungen des Gesundheitswesens
}

\author{
Vorstand der Akademie für Ethik in der Medizin e.V.
}

(C) Die Autor(en) 2010. Dieser Artikel ist auf Springerlink.com mit Open Access verfügbar.

Ethikberatung ist ein in Deutschland relativ neuer Ansatz zur Verbesserung der Qualität der Versorgung von kranken und pflegebedürftigen Menschen. Entsprechende Strukturen wie z. B. Ethik-Komitees, Ethik-Konsile oder Ethik-Foren werden von immer mehr Krankenhäusern sowie Alten- und Pflegeeinrichtungen eingerichtet. Auch bei der Zertifizierung von Gesundheitseinrichtungen wird Ethikberatung zunehmend als ein wichtiges Qualitätskriterium nachgefragt.

Vor dem Hintergrund der wachsenden Bedeutung von Ethikberatung hat die Arbeitsgruppe „Ethikberatung im Krankenhaus“ in der Akademie für Ethik in der Medizin (AEM) die nachfolgenden Standards für Ethikberatung in Einrichtungen des Gesundheitswesens entwickelt. Sie beschreiben die Qualitätskriterien und Basisanforderungen, denen jede Form der Ethikberatung genügen sollte. Die Standards wurden vom Vorstand der AEM am 24. Februar 2010 verabschiedet.

\section{Ziele und Aufgaben}

Ethikberatung dient der Information, Orientierung und Beratung der verschiedenen an der Versorgung beteiligten bzw. davon betroffenen Personen (z. B. Mitarbeitende und Leitung der Einrichtung, Patienten/Bewohner, deren Angehörige und Stellvertreter). Zum Gelingen dieses Beratungsangebots sind klare Ziele und Aufgaben erforderlich.

Allgemeine Ziele von Ethik in Einrichtungen des Gesundheitswesens sind:

- die Sensibilisierung für ethische Fragestellungen,

- die Vermittlung von medizin- und pflegeethischem Wissen,

- die Erhöhung der Kompetenz im Umgang mit ethischen Problemen und Konflikten.

Akademie für Ethik in der Medizin e. V. $(\square)$

Humboldtallee 36, 37073 Göttingen, Deutschland

Tel.: +49-551-399680

E-Mail: vorstand@aem-online.de 
Spezifische Ziele von Ethikberatung in Einrichtungen des Gesundheitswesens sind:

- die Unterstützung eines strukturierten Vorgehens bei ethischen Konflikten,

- die Verbesserung der Sprachfähigkeit und kommunikativen Kompetenz bei ethischen Konflikten,

- die systematische Reflexion über ethische Fragestellungen und Konflikte,

- die Umsetzung allgemeiner moralischer Werte (z. B. Menschenwürde, Autonomie, Verantwortung, Fürsorge, Vertrauen) und spezifischer Werte der jeweiligen Einrichtung, die u. a. in Leitbildern und professionsspezifischen Traditionen verkörpert sind, in reflektiertes Handeln,

- Lösungswege bei Konflikten zwischen unterschiedlichen individuellen und/oder institutionell gefassten Werten und Moralvorstellungen zu suchen und durch gemeinsame Reflexion zu tragfähigen Entscheidungen zu gelangen und diese umzusetzen.

Ziel ist es letztlich, Entscheidungsprozesse hinsichtlich ihrer ethischen Anteile transparent zu gestalten und an moralisch akzeptablen Kriterien auszurichten, d.h. ,gute Entscheidungen“ in ,guten Entscheidungsprozessen“ zu treffen. Dabei zielt Ethikberatung auf die Stärkung der ethischen Kompetenz vor Ort. Sie trägt zur Qualitätssicherung in der Versorgung von Patienten/Bewohnern bei.

Die Aufgaben der Ethikberatung in Einrichtungen des Gesundheitswesens, deren Umsetzung in Abschnitt 3 beschrieben wird, umfassen u. a.:

- die Durchführung individueller ethischer Fallbesprechungen (Ethik-Fallberatung),

- die Erstellung von internen Leitlinien bzw. Empfehlungen (Ethik-Leitlinien) sowie

- die Organisation von internen und öffentlichen Veranstaltungen zu medizin- und pflegeethischen Themen (Ethik-Fortbildung).

\section{Implementierung und Organisation}

a) Verhältnis zu Leitungsebene und Institution

Voraussetzungen für eine erfolgreiche Ethikberatung sind die Verankerung in der Mitarbeiterschaft und die Unterstützung durch die Leitungsebene (Top-down- und Bottom-up-Prinzip). So erfolgt eine Berufung der Mitglieder eines Gremiums zur Ethikberatung, wie z.B. eines Ethik-Komitees, durch die Leitungsebene. In diesem Zusammenhang sind Dienstzeitregelungen, Ausstattung und Budget zu klären. Einrichtungsinterne Leitlinien, die von der Ethikberatung erarbeitet wurden, müssen durch die Leitungsebene bestätigt und umgesetzt werden.

Ethikberatung ist in ihren Inhalten und in der Gestaltung des vereinbarten Vorgehens nicht weisungsgebunden. Ethikberatung ist aber Teil der Organisation und handelt folglich in einem strukturellen Kontext. Ethikberatung berücksichtigt den strukturellen Rahmen und gestaltet diesen durch ihre spezifische Dienstleistung mit. Deshalb arbeitet Ethikberatung - nach einer differenzierenden Analyse - auf eine Integration in die Abläufe und Entscheidungsstrukturen in der Einrichtung hin. Zugleich bleibt sie (qua Auftrag und Geschäftsordnung) ein kritisches Gegenüber und steuert zu konfliktbeladenen, individuellen Verläufen eine relevante Außenperspektive bei. Es ist eine besondere Herausforderung der Ethikberatung, eine angemessene Balance zwischen institutioneller Einbindung und Unabhängigkeit herzustellen. 
Leitung und Mitarbeiter der Einrichtung werden regelmäßig über die Aktivitäten der Ethikberatung informiert.

b) Strukturelle und personelle Voraussetzungen

Für ein Gremium zur Ethikberatung ist eine Satzung und/oder Geschäftsordnung erforderlich. Die Satzung regelt die Bezeichnung des Gremiums, seine inhaltliche Unabhängigkeit, die Ziele und Aufgaben (s. o.), seine Zusammensetzung, den Modus der Berufung, Berichtsund Dokumentationspflichten (s. u.) und die Struktur des Gremiums.

Die Zusammensetzung des Gremiums ist geeignet, die Zielsetzung zu erreichen und die Aufgaben angemessen zu erfüllen. Das Gremium ist multiprofessionell besetzt und besteht je nach Größe der Einrichtung - aus 5 bis 20 Mitgliedern aus verschiedenen Bereichen und Hierarchieebenen. Erforderlich sind Mitglieder mit ärztlicher, pflegerischer und medizinbzw. pflegeethischer Ausbildung. Anzustreben ist darüber hinaus die Mitgliedschaft von Menschen mit einem juristischen, seelsorgerlichen/religiösen, psycho-sozialen und administrativen beruflichen Hintergrund. Wünschenswert ist zusätzlich eine Patienten- bzw. Bewohnerperspektive, die durch Patienten- oder Heimfürsprecher, Mitglieder von Selbsthilfegruppen, die Krankenhaushilfe oder durch engagierte Bürger eingenommen wird.

Erforderliche Qualifikationen für Mitglieder eines Gremiums zur Ethikberatung sind kommunikative und diskursive Kompetenzen, zeitliche Verfügbarkeit und eine entsprechende Weiterbildung. ${ }^{1}$

Die Berufung der Mitglieder erfolgt durch die Leitung der Einrichtung für eine Amtsperiode von mindestens zwei Jahren. Da personelle Kontinuität, Vertrauen und praktische Erfahrung wichtig für den Erfolg von Ethikberatung sind, sollte eine erneute Berufung möglich sein.

Das Gremium wählt einen Vorsitz/Vorstand, der Sitzungstermine festlegt, zu Sitzungen einlädt, eine Tagesordnung erstellt, die Sitzungen leitet, das Budget verwaltet und das Gremium nach außen vertritt.

\section{Umsetzung und Ausgestaltung der Aufgaben}

Ethikberatung identifiziert Probleme und Konflikte in einer Einrichtung und trägt dazu bei, diese Probleme und Konflikte möglichst einvernehmlich zu lösen und die erarbeitete Lösung praktisch umzusetzen. Dies gilt für alle im Folgenden näher spezifizierten Aufgaben.

a) Durchführung individueller ethischer Fallbesprechungen (Ethik-Fallberatung)

Ethik-Fallberatungen dienen der Unterstützung in schwierigen Entscheidungs- bzw. Behandlungssituationen. Sie können von allen an der Entscheidung bzw. Behandlung Beteiligten beantragt werden (z.B. Mitarbeitende aus den verschiedenen Berufsgruppen, Patienten/ Bewohner, deren Angehörige und Stellvertreter). Grundsätzlich ist zwischen Einzelberatungen (z. B. bei individueller Gewissensnot) und gemeinsamen Fallbesprechungen auf der Station bzw. im Wohnbereich zu unterscheiden. Bei gemeinsamen Fallbesprechungen ist

\footnotetext{
1 Vgl.: Simon A, May A, Neitzke G (2005) Curriculum „Ethikberatung im Krankenhaus“. Ethik Med 17:322-326; Bockenheimer-Lucius G, May AT (2007) Ethikberatung - Ethik-Komitee in Einrichtungen der stationären Altenhilfe (EKA). Eckpunkte für ein Curriculum. Ethik Med 19:331-339 (beide Curricula können unter www.ethikkomitee.de eingesehen und heruntergeladen werden).
} 
zu klären, wer an der Beratung teilnehmen soll und wer über diese zu informieren ist. Eine Einbeziehung des Patienten/Bewohners ist anzustreben. Darüber hinaus sollte geregelt sein, in welchen Fällen eine Zustimmung des Patienten/Bewohners zur Ethik-Fallberatung einzuholen ist.

Die Ethik-Fallberatung findet in einem geschützten, störungsfreien Rahmen statt. Die gesetzlichen Bestimmungen zum Datenschutz und zur Einhaltung der Schweigepflicht sind zu beachten.

Das Beratungsgespräch wird von einem Mitglied des Gremiums zur Ethikberatung moderiert; die Orientierung an einem Moderationsleitfaden ist sinnvoll. Da die Ethik-Fallberatung ein Prozess ist, sind Folgeberatungen möglich. Bei der organisatorischen Planung ist die Zeit für die Durchführung sowie für die Vor- und Nachbereitung der Ethik-Fallberatung zu berücksichtigen.

Bei einer Ethik-Fallberatung verbindet sich Moderationskompetenz mit ethischer Expertise. Aufgabe der Berater ist es einerseits, alle für die Bewertung des Falles erforderlichen Details sichtbar zu machen und allen Anwesenden Raum zur Beteiligung zu geben, andererseits die ethischen Fragen herauszuarbeiten und die Möglichkeiten des weiteren Vorgehens nach ethischen Kriterien zu gewichten. Für das weitere Vorgehen sind die theoretischen Aspekte und die realen Gegebenheiten abzuwägen, so dass die Verantwortlichen das weitere Vorgehen festlegen und in Handlung umsetzen können. Ein Konsens ist anzustreben; dieser ist erreicht, wenn alle an der Fallberatung Beteiligten die vorgeschlagene Lösung mittragen und gemeinsam verantworten können.

Das Ergebnis einer Fallberatung wird dokumentiert.

b) Erstellung von internen Leitlinien bzw. Empfehlungen (Ethik-Leitlinien)

Ethik-Leitlinien sind Handlungsempfehlungen, die sich aus immer wiederkehrenden Situationen (z. B. Umgang mit Patientenverfügungen, Legen einer PEG-Sonde, Wiederbelebung, Therapiezieländerung, Umgang mit Zeugen Jehovas) ableiten und die als Orientierungshilfe für Einzelfallentscheidungen dienen. Ihre Erarbeitung richtet sich thematisch nach dem in der Einrichtung bestehenden Bedarf.

Ethik-Leitlinien werden durch Mitglieder des Gremiums zur Ethikberatung themenbezogen unter Einbeziehung von sachkundigen Personen aus der Einrichtung oder von außerhalb erarbeitet. Sie müssen den gesetzlichen Vorschriften sowie dem wissenschaftlichen Standard entsprechen und daher regelmäßig aktualisiert werden.

Ethik-Leitlinien werden von der Leitung der Einrichtung verabschiedet.

c) Organisation von Veranstaltungen zu medizin- und pflegeethischen Themen (Ethik-Fortbildung)

Ethik-Fortbildungen dienen der Sensibilisierung für ethische Fragestellungen, der Vermittlung von ethischem Wissen und der Erhöhung der Kompetenz im Umgang mit ethischen Problemen und Konflikten. Zielgruppen sind u. a. die Mitarbeitenden der Einrichtung, die Patienten/Bewohner und deren Angehörige sowie die interessierte Öffentlichkeit.

Das Gremium zur Ethikberatung klärt in Zusammenarbeit mit den Verantwortlichen für die Innerbetriebliche Fortbildung oder einer ggf. vorhandenen Bildungseinrichtung den Bedarf an Ethik-Fortbildungen hinsichtlich der erforderlichen Inhalte und Methoden und regelt bzw. koordiniert deren Organisation. Die Durchführung kann durch Mitglieder der Ethikberatung oder durch andere geeignete Personen (z.B. andere Mitarbeitende der Einrichtung, externe Experten) erfolgen. 
Das Spektrum von Ethik-Fortbildungen kann von themenbezogenen Teambesprechungen bis hin zu öffentlichen Veranstaltungen (z.B. Ethik-Tag, Patientenforum) reichen.

\section{Dokumentation und Evaluation}

Die verschiedenen Aktivitäten der Ethikberatung werden in geeigneter Weise dokumentiert und zum Zweck der Qualitätssicherung fortlaufend evaluiert. Ein regelmäßiger (z. B. jährlicher) Tätigkeitsbericht wird erstellt, der Leitung und den Mitarbeitern bekannt gemacht und mit diesen diskutiert.

Die Ergebnisse einer Ethik-Fallberatung, die konkrete Auswirkungen auf die weitere Behandlung oder Betreuung des Patienten/Bewohners haben, sind schriftlich in den Krankenunterlagen zu dokumentieren. Eine Kopie der Dokumentation wird unter Berücksichtigung datenschutzrechtlicher Vorgaben vom Gremium zur Ethikberatung zur Absicherung der an der Besprechung Beteiligten sowie für die Evaluation aufbewahrt.

Die Dokumentation der Ethik-Fallberatung sollte sich weitgehend auf einen Ergebnisbericht beschränken, wobei der Schwerpunkt auf der ethischen Begründung für die gewählte Handlungsoption bzw. Vorgehensweise liegt.

Im Sinne der Qualitätssicherung von Ethikberatung empfiehlt es sich, das Ergebnis einer Ethik-Fallberatung - z. B. in der nächsten Sitzung des Gremiums zur Ethikberatung nachzubesprechen.

Open Access Dieser Artikel unterliegt den Bedingungen der Creative Commons Attribution Noncommercial License. Dadurch sind die nichtkommerzielle Nutzung, Verteilung und Reproduktion erlaubt, sofern der/die Originalautor/en und die Quelle angegeben sind. 